\title{
Conformational preferences of the substrates of lactate dehydrogenase
}

\author{
R.K. Schmidt, J.E. Gready* \\ Division of Biochemistry and Molecular Biology, John Curtin School of Medical Research, Australian National University, G.P.O. Box 334, \\ Canberra ACT 2601, Australia
}

Received 4 January 1999; accepted 22 June 1999

\begin{abstract}
The substrates of lactate dehydrogenase (pyruvate and lactate) as well as an inhibitor (oxamate) are characterized using ab initio methods employing the basis sets $6-31+\mathrm{G}(\mathrm{d})$ and $6-31+\mathrm{G}(\mathrm{d}, \mathrm{p})$ at both RHF and MP2 levels. The first aim of these studies is to explore the conformational preferences of these molecules and to compare the minimum energy conformation with that found in crystallographic structures of oxamate bound in the enzyme active site. In the lowest energy conformer of pyruvate, the carboxyl group is rotated about $80^{\circ}$ from the plane defined by the carbonyl group and methyl carbon atom, a preference dominated by repulsion between the carboxyl and carbonyl oxygens. A relatively small barrier of $\sim 2 \mathrm{kcal} / \mathrm{mol}$ for rotation of the carboxyl group is calculated at the MP2/6-31+G(d,p) level. In contrast, both oxamate and lactate prefer a nearly planar orientation of the carboxyl group, which is more like the enzyme-bound conformation of oxamate. In these molecules, the repulsion between the oxygen atoms is counterbalanced by intramolecular hydrogen bonds, which are maximized in a nearplanar conformation. The barrier to carboxyl rotation in oxamate is $\sim 4 \mathrm{kcal} / \mathrm{mol}$ at the MP2/6-31+G(d, p) level, while that for lactate is much higher, about $10 \mathrm{kcal} / \mathrm{mol}$ at the $\mathrm{RHF} / 6-31+\mathrm{G}(\mathrm{d})$ level. Thus, even though the minimum-energy lactate conformation is more similar to the enzyme-bound oxamate conformation than that for pyruvate, a steeper conformational energy surface indicates that at least as much energy would be required for the smaller torsional changes which occur on binding.

A second aim of these studies is to evaluate whether the semiempirical methods AM1 and PM3 commonly used in QM/MM calculations of enzyme reactions adequately reproduce higher-level ab initio conformational results. The AM1 method is shown to be accurate for pyruvate and lower-energy regions of lactate, but less accurate for oxamate. PM3 tends to overestimate barriers to conformational changes for these molecules. (C) 2000 Elsevier Science B.V. All rights reserved.
\end{abstract}

Keywords: Lactate dehydrogenase; Pyruvate; Lactate; Oxamate; Ab initio; Semiempirical; Conformer

\section{Introduction}

Pyruvate and L-lactate are primary metabolic intermediates. Pyruvate is formed from the decomposition of glucose in glycolysis and, under aerobic conditions,

\footnotetext{
* Corresponding author. Tel.: + 61-2-6279-8304; fax: + 61-26249-0415.

E-mail address: jill.gready@anu.edu.au (J.E. Gready).
}

is then shuttled into the citric acid cycle. When oxygen is not available, pyruvate is instead reduced to lactate in the Cori cycle by the enzyme L-lactate dehydrogenase (LDH), coupled with oxidation of the coenzyme NADH in the following reaction:

$$
\begin{aligned}
& \mathrm{CH}_{3} \mathrm{C}(=\mathrm{O}) \mathrm{COO}^{-}+\mathrm{NADH}+\mathrm{H}^{+} \\
& \rightleftharpoons \mathrm{CH}_{3} \mathrm{CH}(-\mathrm{OH}) \mathrm{COO}^{-}+\mathrm{NAD}^{+}
\end{aligned}
$$


(a) P1

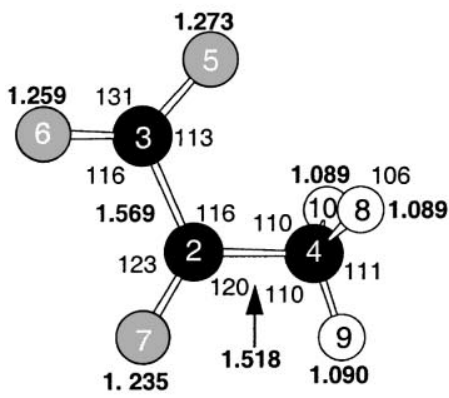

O6-C3-C2-O7

$\mathrm{O} 5-\mathrm{C} 3-\mathrm{C} 2-\mathrm{O} 7$

$\mathrm{H} 9-\mathrm{C} 4-\mathrm{C} 2-\mathrm{O} 7$

0.0

0.0

(c) P3

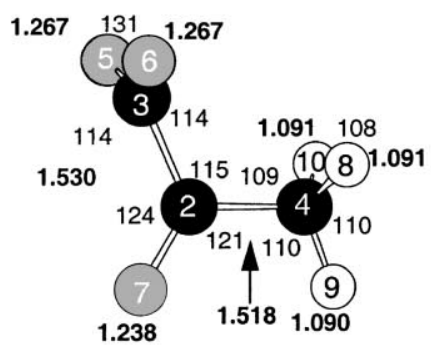

(b) P2

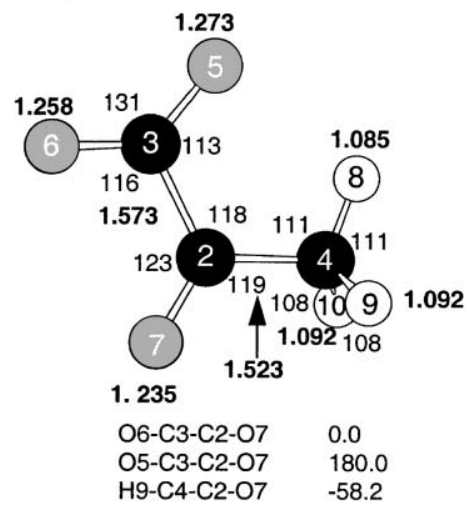

(d) P3alt

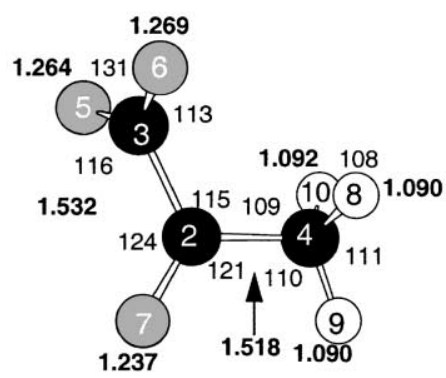

O6-C3-C2-O7

104.6

O5-C3-C2-O7 -77.6

H9-C4-C2-O7 5.0

(e) P4

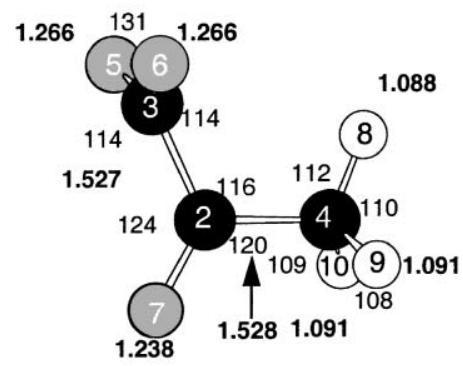

$\begin{array}{ll}\mathrm{O} 6-\mathrm{C} 3-\mathrm{C} 2-\mathrm{O} 7 & 91.5 \\ \mathrm{O} 5-\mathrm{C} 3-\mathrm{C} 2-\mathrm{O} 7 & -91.5 \\ \mathrm{H} 9-\mathrm{C} 4-\mathrm{C} 2-\mathrm{O} 7 & -58.8\end{array}$

Fig. 1. Bond lengths (in bold) and angles for five conformers of pyruvate optimized at the MP2/6-31+G(d, p) level, with selected torsions given below. Carbon, oxygen and hydrogen atoms are coloured black, grey and white, respectively, with the atom numbers referred to in the text superimposed on each atom. 
(a) Ox1

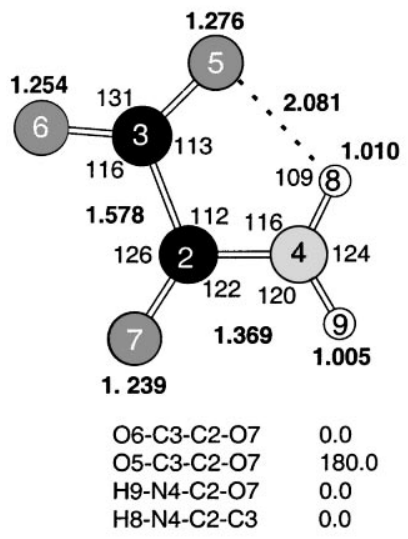

(b) Ox1alt

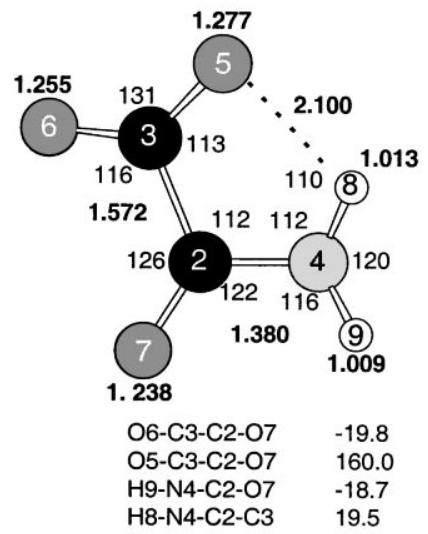

(c) $0 \times 2$

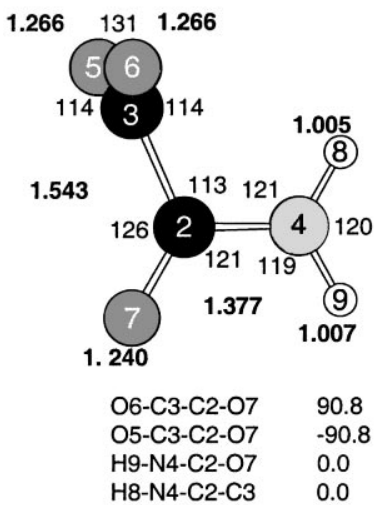

Fig. 2. Bond lengths (in bold) and angles for three conformers of oxamate optimized at the MP2/6-31+G(d, p) level, with selected torsions given below. Carbon, oxygen, hydrogen and nitrogen atoms are coloured black, grey, white and light grey, respectively, with the atom numbers referred to in the text superimposed on each atom.

This reaction regenerates the $\mathrm{NAD}^{+}$required for glycolysis to continue in the anaerobic environment.

While numerous kinetic and mutagenesis studies have characterized the enzyme LDH [1], much is still unknown about the reaction mechanism on an atomic scale. In particular, it is unknown whether the reaction mechanism involves concerted proton and hydride-ion transfers or sequential transfers. Two computational studies from this group suggest that the sequential mechanism is more likely, but they differ in the order in which the proton and hydride ions are transferred. Semiempirical (AM1) studies of fragments in the active site indicate that proton transfer to the substrate precedes hydride transfer [2]. In contrast, hybrid quantum mechanical/ molecular mechanical (QM/MM) calculations in which the full enzyme environment was included indicate that hydride transfer occurs first [3]. Further $\mathrm{QM} / \mathrm{MM}$ calculations are required to optimize the computational protocol and ensure that the results obtained from this relatively new method are physically reliable.

In these QM/MM calculations, all species directly involved in the chemical reaction are modelled quantum mechanically (in this case, minimally, the substrate, NADH, and proton donor His195) while the remaining environment is represented by an empirical molecular mechanical force field. To minimize computational time, the quantum mechanical part of the system employs semiempirical methods 
(a) L1

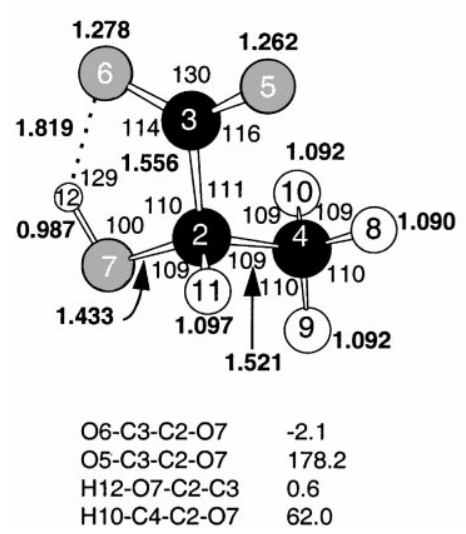

(b) L2

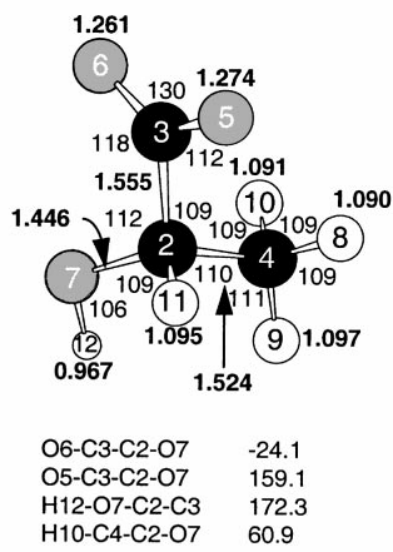

Fig. 3. Bond lengths (in bold) and angles for two conformers of lactate optimized at the MP2/6-31+G(d, p) level, with selected torsions given below. Carbon, oxygen and hydrogen atoms are coloured black, grey and white, respectively, with the atom numbers referred to in the text superimposed on each atom.

(AM1 or PM3), and thus it is important to ensure that these methods represent the system reliably, including reproducing higher level ab initio results reasonably well. Both energies and geometries must be modelled consistently as well as the conformational flexibility of the substrates. The reaction catalyzed by LDH involves the conversion of a planar $\mathrm{sp}^{2}$ carbon to a tetrahedral $\mathrm{sp}^{3}$ carbon, resulting in a shift of the relative orientations of the carboxyl and methyl groups of the substrate within a tightly packed active site. The energetic cost or benefit of these conformational changes, as well as the conformational changes which might occur upon substrate binding, could be significant in determining the reaction mechanism, particularly as the energy surface for proton and hydride-ion transfers calculated previously is relatively shallow and potentially of the same order of magnitude as for the conformational changes.

Here we present ab initio studies of the energy and geometry of several conformations of the substrates of LDH, pyruvate and lactate. Corresponding studies of the inhibitor oxamate, which differs from pyruvate only in the substitution of a methyl group by an amino group, are also included. The anions are studied because the presence of a strong salt bridge between a conserved arginine residue (Arg171 in dogfish muscle LDH [4]) and the carboxyl group in the active site makes it unlikely that the substrates are in the acid form. While most previous studies have focussed on the protonated acid form of these molecules [5-9], earlier studies on the deprotonated anionic form $[8,9]$ did not include diffuse functions. As diffuse functions are generally necessary to represent anions accurately [10], we have included them in these computations. $\mathrm{Ab}$ initio results are compared with those obtained using AM1 and PM3 methods to evaluate whether it is reasonable to treat the reaction using these methods in hybrid QM/MM studies.

\section{Methods}

Two-dimensional projections of the potential energy surface were first generated from RHF calculations with the $6-31+G(d)$ basis set for pyruvate, oxamate, and lactate. In these conformational energy scans, two torsional degrees of freedom were varied in $30^{\circ}$ increments and geometry optimization performed at each grid point. For pyruvate, the degrees of freedom were the methyl and carboxyl group torsions; for oxamate, the amino and carboxyl group torsions; and for lactate, the hydroxyl and carboxyl group torsions. These surfaces were used to identify significant conformers and to estimate barriers to conversion among conformers. The conformers chosen for further study, as illustrated in Figs. 1-3, were then optimized 
Table 1

Energies (in $\mathrm{kcal} / \mathrm{mol}$ relative to P3) for selected conformers of pyruvate

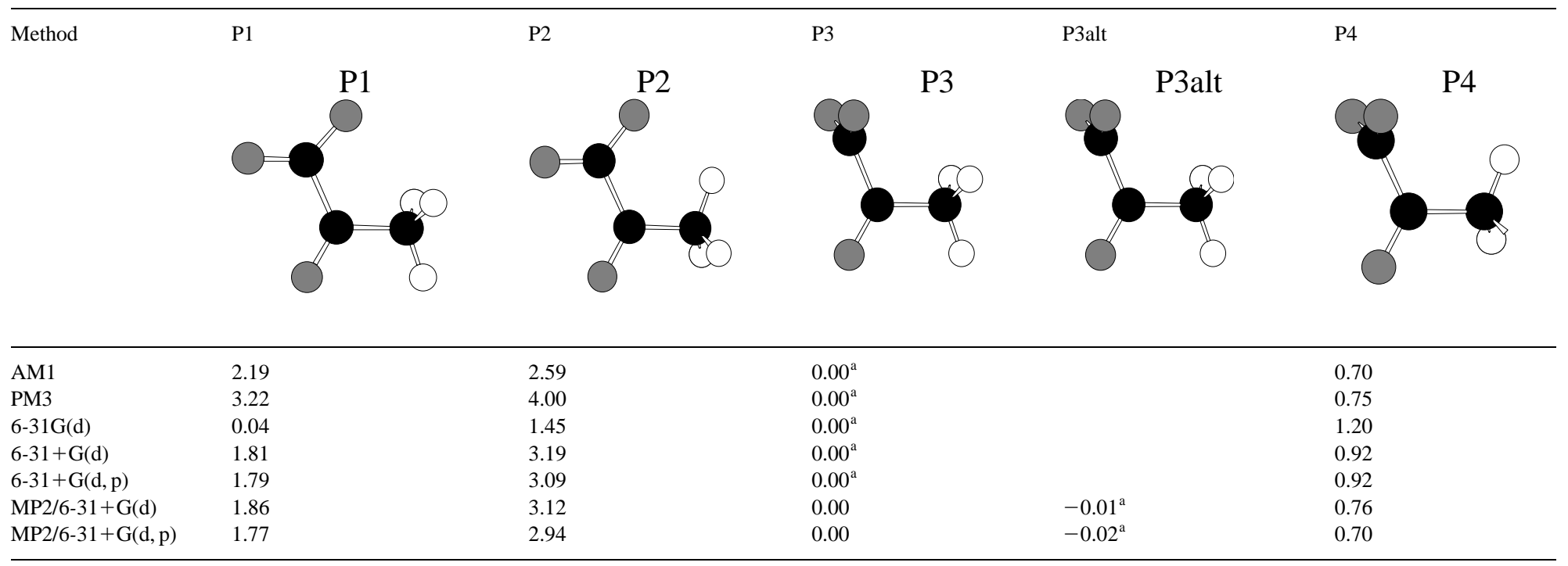

${ }^{a}$ This conformer is a true minimum with no imaginary frequencies. All other conformers in the table have at least one imaginary frequency. 
using the basis sets $6-31+\mathrm{G}(\mathrm{d})$ and $6-31+\mathrm{G}(\mathrm{d}, \mathrm{p})$ at both RHF and MP2 levels. For pyruvate, the conformers were also optimized at the 6-31G(d) basis set level for comparison but, as discussed later, it was clear that the study of these anions requires diffuse functions [10]. Grid scans and geometry optimizations were also performed for the three molecules using the semiempirical methods AM1 and PM3. Vibrational frequencies were calculated for conformers to distinguish between minima (with real frequencies) and saddle points (with one or more imaginary frequencies). All calculations were performed using GAUSSIAN94 [11] on Fujitsu VPP300 or Silicon Graphics PowerChallenge computers.

In the following, the molecular plane is defined by the three carbon atoms in pyruvate and lactate $(\mathrm{C} 2, \mathrm{C} 3$ and $\mathrm{C} 4$ ) and the nitrogen and two carbon atoms in oxamate $(\mathrm{C} 2, \mathrm{C} 3$ and $\mathrm{N} 4)$. The barriers to torsional rotation for pyruvate are calculated as the average of differences among several conformers. For example, the barrier to carboxyl rotation is calculated as the average of the difference in energy between the planar (P1) and perpendicular (P3) conformations (see Fig. 1) for pairs of conformers with either eclipsed or staggered methyl conformations, so that $E$ (barrier) $=$ $1 / 2 \times((E(\mathrm{P} 1)-E(\mathrm{P} 3))+(E(\mathrm{P} 2)-E(\mathrm{P} 4))$.

\section{Results}

\subsection{Pyruvate}

Pyruvate has two conformational degrees of freedom: rotation of the methyl group and the carboxyl group. The $6-31+\mathrm{G}(\mathrm{d})$ conformational scan for pyruvate was characterized as a shallow potential energy landscape, with methyl group rotation occurring more easily than carboxyl group rotation. As shown in Fig. 1 , four conformations of pyruvate (P1, P2, P3 and P4) were chosen for further study. In these conformations, the methyl group is either staggered or eclipsed with respect to the carbonyl group, and the carboxyl group is either lying in or perpendicular to the molecular plane. As can be seen in Table 1, the methyl group prefers to be eclipsed with respect to the carbonyl group, although rotation to the staggered form requires only about $1 \mathrm{kcal} / \mathrm{mol}$. Rotation of the carboxyl group, however, requires more energy. For all basis sets except 6-31G(d) (for which the results are qualitatively different from those for all other methods), the perpendicular conformation in which the carboxyl group is rotated about $90^{\circ}$ out of the molecular plane is more stable than the planar conformation by about $2 \mathrm{kcal} / \mathrm{mol}$. For most calculations, this perpendicular conformation with a staggered methyl group (P3) is the global minimum, and the molecule is symmetrical with respect to the molecular plane. After including electron correlation, however, the global minimum is a pair of enantiomers with the carboxyl group rotated about $10^{\circ}$ from perpendicular. This conformer (P3alt) is less than $0.02 \mathrm{kcal} / \mathrm{mol}$ lower in energy than the symmetrical conformation P3.

Fig. 1 shows the geometries of all five conformers optimized at the highest level of theory (MP2/6-31+G(d,p)). When the carboxyl group is planar, the repulsion between the carbonyl oxygen O7 and the carboxyl oxygen O6 causes the carboxyl group to tilt so that the angle $\mathrm{C} 2-\mathrm{C} 3-\mathrm{O} 6$ increases to $116^{\circ}$ and the $\mathrm{C} 2-\mathrm{C} 3-\mathrm{O} 5$ angle decreases to $113^{\circ}$. The carboxyl C3-O6 bond (nearest the carbonyl group) is slightly shorter $(0.014 \AA)$ than the carboxyl C3-O5 bond (farthest from the carbonyl group). For the symmetrical perpendicular conformation (P3), both $\mathrm{C} 2-\mathrm{C} 3-\mathrm{O} 6$ and $\mathrm{C} 2-\mathrm{C} 3-\mathrm{O} 5$ angles are $114^{\circ}$ and C3-O5 and C3-O6 distances are $1.267 \AA$, while for the near-perpendicular minimum (P3alt) the geometry is almost as symmetrical. The $\mathrm{C} 2-\mathrm{C} 3$ bond length is longer for the planar (average 1.571 $\AA$ ) compared with the perpendicular (average $1.528 \AA$ ) conformation. This lengthening, along with the tilting of the carboxyl group, allows the planar conformations to maximize the distance between the oxygens $\mathrm{O} 7$ and O6, which are strongly negatively charged according to Mulliken charge analysis. In all five conformers, the partial charge of the carbonyl oxygen $\mathrm{O} 7$ is near the average -0.56 , while that for the carboxyl oxygens $\mathrm{O} 5$ and $\mathrm{O} 6$ ranges from -0.72 to -0.75 .

The AM1 method reproduces the relative energies of the conformers well, compared with the best calculation (MP2/6-31+G(d,p)). The AM1 predictions for the average barrier (see Section 2) to methyl $(0.55 \mathrm{kcal} / \mathrm{mol})$ and carboxyl $(2.04 \mathrm{kcal} / \mathrm{mol})$ group rotations compare favourably with those found using the best ab initio method $(0.94$ and $2.01 \mathrm{kcal} / \mathrm{mol}$, respectively). The PM3 method also predicts the 
Table 2

Energies (in $\mathrm{kcal} / \mathrm{mol}$ relative to Ox1alt) for selected conformers of oxamate

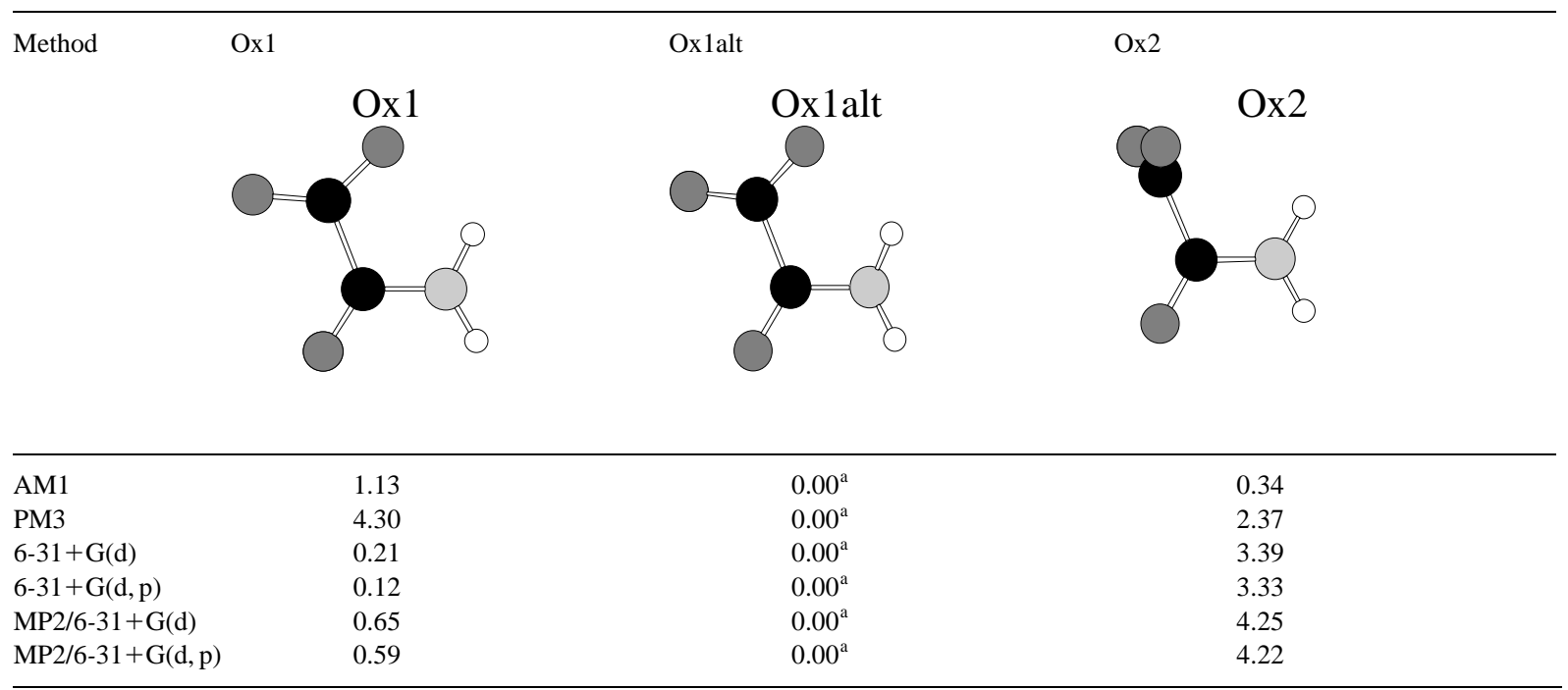

${ }^{a}$ This conformer is a true minimum with no imaginary frequencies. All other conformers in the table have at least one imaginary frequency.

relative ordering of the conformers correctly, but in this case while the barrier to methyl group rotation $(0.77 \mathrm{kcal} / \mathrm{mol})$ is even closer to the ab initio result, the barrier to carboxyl group rotation is overestimated by about $1 \mathrm{kcal} / \mathrm{mol}(3.24 \mathrm{kcal} / \mathrm{mol})$. Both semiempirical methods found the global minimum to be the symmetrical P3 conformer instead of P3alt.

\subsection{Oxamate}

Oxamate has two conformational degrees of freedom, rotation of the carboxyl group and the amino group. The $6-31+\mathrm{G}(\mathrm{d})$ conformational scan in which the amino group was constrained to be planar, indicated that the surface was much steeper than that found for pyruvate, with barriers greater than $25 \mathrm{kcal} /$ mol for rotation of the amino group. Barriers to rotation about the carboxyl group are much lower, with an energy difference of about $3 \mathrm{kcal} / \mathrm{mol}$ between $\mathrm{Ox} 1$ (in which the carboxyl group is planar, Fig. 2) and Ox2 (in which the carboxyl group is perpendicular to the molecular plane). In contrast to pyruvate, it is the planar conformation Ox 1 which is lower in energy, presumably because of the hydrogen bond between $\mathrm{O} 5$ and $\mathrm{H} 8$ which is not possible for the perpendicular conformation Ox2. These two conformers were further studied at higher basis set levels
(Table 2). Little difference was observed between $6-31+\mathrm{G}(\mathrm{d})$ and $6-31+\mathrm{G}(\mathrm{d}, \mathrm{p})$ basis set results, while adding correlation increased the difference in energy between the two conformers slightly ( $\sim 0.3 \mathrm{kcal} / \mathrm{mol})$. Conformer Ox1 is not a true minimum, however, and possesses one imaginary frequency. Instead, the global minima are an enantiomeric pair of molecules (Ox1alt) in which the amino group is moderately tetrahedral with hydrogens extending out on the same side of the molecular plane, while the carboxyl group is rotated approximately $20^{\circ}$ from this plane. Conformer Ox1alt ranging from 0.1 to $0.7 \mathrm{kcal} / \mathrm{mol}$ is more stable than the planar conformer Ox 1 , so the energy surface is very shallow for this moderate deformation of the carboxyl group torsion. Conformer Ox2 is a saddle point with two imaginary frequencies, and all optimizations which started with the same perpendicular carboxyl group but with a tetrahedral amino group resulted in the global minimum Ox1alt. Note, however, that on the $6-31+\mathrm{G}(\mathrm{d})$ conformational energy surface, when the amino group is constrained to be planar and $90^{\circ}$ out of the molecular plane so that the intramolecular hydrogen bond is not possible, the carboxyl group prefers to be out-of-plane as well, emphasizing that the hydrogen bond is mainly responsible for stabilizing the planar conformation.

Fig. 2 shows the geometries determined for the 
Table 3

Energies (in $\mathrm{kcal} / \mathrm{mol}$ relative to L1) for selected conformers of lactate (both conformers at all levels of theory are true minima with no imaginary frequencies)

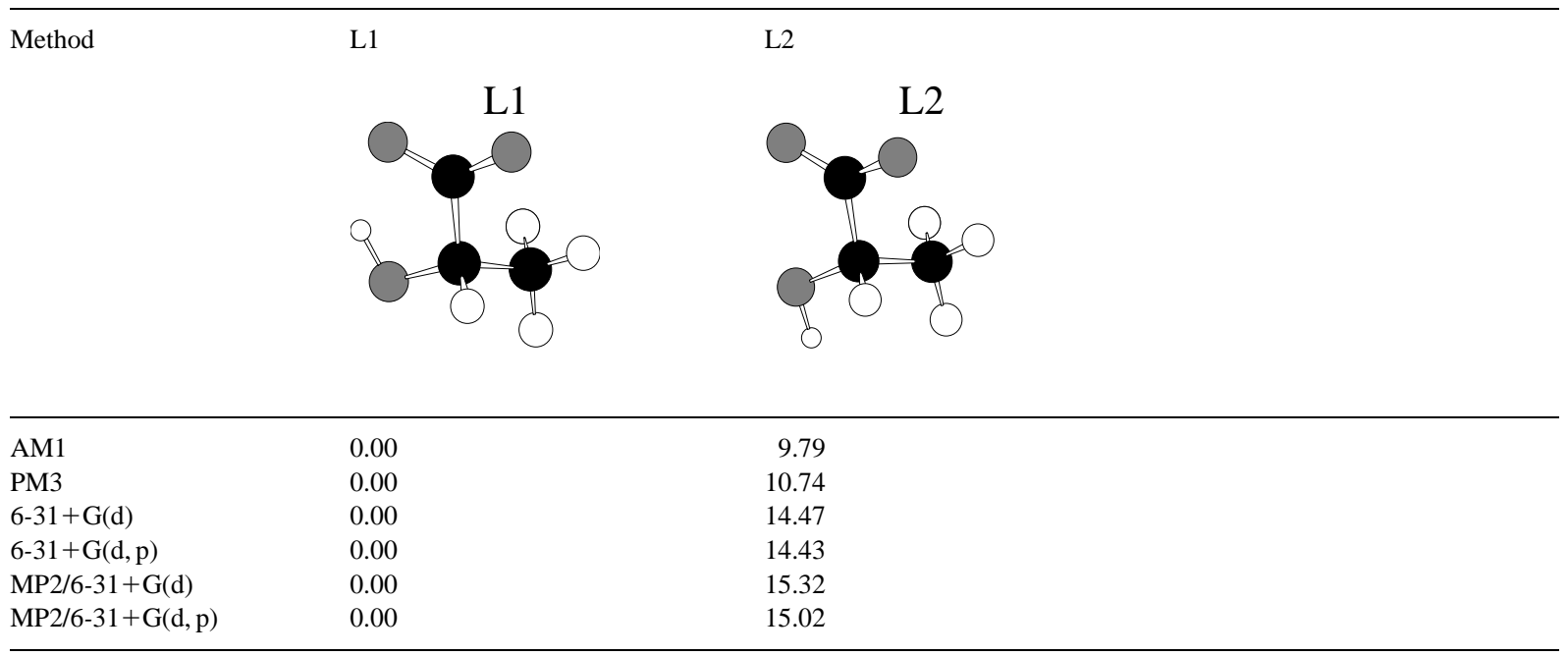

three conformers optimized using MP2/6-31+G(d, p). As for pyruvate, in the planar Ox1 or near-planar Ox1alt conformers the repulsion between O6 and O7 causes a tilting of the carboxyl group away from the carbonyl group, with the $\mathrm{O} 6-\mathrm{C} 3-\mathrm{C} 2$ angle $\left(116^{\circ}\right)$ larger than the $\mathrm{O} 5-\mathrm{C} 3-\mathrm{C} 2$ angle $\left(113^{\circ}\right)$, and the $\mathrm{C} 2-$ C3 bond $\sim 0.03 \AA$ longer for the Ox1 and Ox1alt conformers compared with the Ox2 conformer. The $\mathrm{C} 3-\mathrm{O} 6$ and $\mathrm{C} 3-\mathrm{O} 5$ bond lengths are symmetrical only in the perpendicular conformation (both being $1.266 \AA$ ) while the $\mathrm{C} 3-\mathrm{O} 5$ bond is about $0.02 \AA$ longer than the $\mathrm{C} 3-\mathrm{O} 6$ bond in the Ox1 and Ox1alt conformations due to the hydrogen bond between $\mathrm{O} 5$ and $\mathrm{H} 8$. This hydrogen-bonded $\mathrm{O} 5-\mathrm{H} 8$ distance is about $2.1 \AA$ and the O5-H8-N4 angle is about $109^{\circ}$. In these conformations, this favourable hydrogen bond counterbalances the repulsion between the electron-rich carboxyl oxygen O6 and the carbonyl oxygen $\mathrm{O} 7$, for which the partial Mulliken charge in all conformers is near the average -0.68 . In the Ox 1 and Ox1alt conformers, the carboxyl oxygen O5 involved in the hydrogen bond has a higher charge $(-0.80)$ compared with the carboxyl oxygen O6 $(-0.71)$.

The AM1 method is not as successful at replicating the $a b$ initio results for oxamate as for pyruvate, and the geometry of the minimum energy conformation is quite different. The tetrahedral amino group confor- mation in the AM1 minimum structure is similar to the Ox1alt conformation, with an $\mathrm{H} 9-\mathrm{N} 4-\mathrm{C} 2-\mathrm{O} 7$ torsion of -13.8 and an $\mathrm{H} 8-\mathrm{N} 4-\mathrm{C} 2-\mathrm{C} 3$ torsion of 18.9 , but the carboxyl group torsion $\mathrm{O} 6-\mathrm{C} 3-\mathrm{C} 2-\mathrm{O} 7$ is quite different $\left(-59.4^{\circ}\right.$ instead of $-19.8^{\circ}$ for MP2/6-31+G(d,p)). Because of this large discrepancy in the carboxyl group torsion for the AM1 minimum, which makes the molecule more nearly perpendicular than planar, it is not surprising that the relative energies of the three conformers are different. The perpendicular conformation Ox2 is only slightly higher in energy than the minimum conformation Ox1alt according to the AM1 results $(0.34 \mathrm{kcal} / \mathrm{mol})$ while the planar $\mathrm{Ox} 1$ conformation is $1.13 \mathrm{kcal} / \mathrm{mol}$ higher in energy. The PM3 results follow this same ordering but with higher energy differences $(2.37$ and $4.30 \mathrm{kcal} / \mathrm{mol}$, respectively) and predict a carboxyl group orientation which is rotated even closer to the perpendicular conformation with an $\mathrm{O} 6-\mathrm{C} 3-\mathrm{C} 2-\mathrm{O} 7$ torsion of $-73.7^{\circ}$.

\subsection{Lactate}

With three conformational degrees of freedom (carboxyl, hydroxyl, and methyl group rotations), the potential energy surface for lactate is more complex than those for pyruvate or oxamate. Two conformational energy scans were performed, in 
which the methyl group was fixed to be either staggered or eclipsed with respect to the hydroxyl group, while the carboxyl and hydroxyl group torsions were varied. The topologies of the resulting potential energy surfaces are very similar except that the surface with the eclipsed methyl group is about $3.7 \mathrm{kcal} / \mathrm{mol}$ higher in energy at each point than for a staggered methyl group.

Two similar minima were found on both the eclipsed and staggered methyl group maps. In the global minimum $\mathrm{L} 1$, the atoms $\mathrm{O} 6, \mathrm{C} 3, \mathrm{C} 2, \mathrm{O} 7$, and $\mathrm{H} 12$ are very close to coplanar, and a hydrogen bond is observed between $\mathrm{O} 6$ and H12. Rotation of the carboxyl and hydroxyl groups is hindered by relatively high barriers. If the hydroxyl group torsion $\mathrm{H} 12-\mathrm{O} 7-\mathrm{C} 2-\mathrm{C} 3$ is fixed to the value found in $\mathrm{L} 1$ $\left(\sim 0^{\circ}\right)$, the barrier to rotation of the carboxyl group is about $10 \mathrm{kcal} / \mathrm{mol}$ at the RHF/6-31+G(d) level. The maximum error in this barrier due to the neglect of electron correlation is estimated to be about $1 \mathrm{kcal} /$ mol, as the relative energies predicted for oxamate and pyruvate differ by only $0.02-0.86 \mathrm{kcal} / \mathrm{mol}$ when comparing RHF and MP2 results. A barrier of about $15 \mathrm{kcal} / \mathrm{mol}$ is found for rotation of the hydroxyl group when holding the carboxyl group fixed to the approximate position found in L1 (O6-C3-C2$\mathrm{O} 7$ torsion $\sim 0^{\circ}$ ). Despite the large energy cost associated with rotation of the hydroxyl group, a second minimum with no imaginary frequencies does exist in which the hydroxyl group is pointing directly away from the carboxyl group (H12-O7-C2-C3 torsion $\sim 180^{\circ}$ ). In this conformer (L2), the carboxyl group has rotated slightly from the plane defined by $\mathrm{H} 12$, $\mathrm{O} 7$, and $\mathrm{C} 2$, with a torsion $\mathrm{O} 6-\mathrm{C} 3-\mathrm{C} 2-\mathrm{O} 7$ of $-24.1^{\circ}$ at the MP2/6-31+G(d,p) level. As may be seen in Table 3, this second minimum is much higher in energy than the global minimum $(\sim 15 \mathrm{kcal} / \mathrm{mol})$, partly because of the loss of the intramolecular hydrogen bond between $\mathrm{H} 12$ and $\mathrm{O} 6$.

Fig. 3 shows the geometries of the two minima calculated at the MP2/6-31+G(d,p) level. All optimizations which started with an eclipsed methyl group resulted in a staggered methyl group, and, thus, only the two conformers illustrated were studied. The bond lengths and angles in the conformers L1 and L2 are quite similar except for distortions due to the hydrogen bond in L1. The $\mathrm{C} 3-\mathrm{C} 2-\mathrm{O} 7$ and $\mathrm{C} 6-\mathrm{C} 3-\mathrm{C} 2$ angles are compressed about 2 and $4^{\circ}$, respectively, for the L1 conformer compared with L2, and both the $\mathrm{C} 3-\mathrm{O} 6$ and the $\mathrm{O} 7-\mathrm{H} 12$ bonds are lengthened by about $0.02 \AA$ due to the hydrogen bond in the L 1 conformation. Both minima have nearly identical C2-C3 bond lengths $(1.556 \AA)$. The partial charges for $\mathrm{O} 5, \mathrm{O} 6$, and $\mathrm{O} 7$ are $-0.74,-0.80$, and -0.67 for the $\mathrm{L} 1$ conformer, and $-0.77,-0.73$, and -0.57 for the $\mathrm{L} 2$ conformer.

Both the AM1 and PM3 methods predict a similar geometry for the global minimum L1, with the carboxyl and hydroxyl group torsions only slightly greater than found using $\mathrm{MP} 2 / 6-31+\mathrm{G}(\mathrm{d}, \mathrm{p})$ (with averages of $\sim-7$ compared with $-2.1^{\circ}$, and $\sim 9$ compared with $0.6^{\circ}$, respectively). While the lower energy region of the conformational scans are similar, the higher energy regions are different for the semiempirical methods, and the L2 conformation has quite a different carboxyl group torsion than that for MP2/6-31+G(d,p) ( -73 compared with $\left.-24.1^{\circ}\right)$ resulting in a decrease of $5 \mathrm{kcal} / \mathrm{mol}$ in the energy difference between the L1 and L2 conformers.

\section{Discussion}

The results indicate that the conformational preferences of the substrates and inhibitor of LDH depend on a balance of hydrogen bonding interactions and electrostatic repulsion in these electron-rich anions. For pyruvate, no intramolecular hydrogen bonds are possible and the dominant influence on the conformational preference is the repulsion between the carboxyl and carbonyl oxygens. This repulsion is minimized when the carboxyl group is almost perpendicular to the molecular plane in the lowest energy conformer (P3alt). The barrier to carboxyl group rotation is $2.01 \mathrm{kcal} / \mathrm{mol}$ at the MP2/6-31+G(d,p) level. On the contrary, in the isoelectronic pyruvate-analogue oxamate, an intramolecular hydrogen bond is possible in the planar or near-planar conformation, which counterbalances the repulsion between the carboxyl and carbonyl oxygens. Hence, in contrast to pyruvate, a nearly planar carboxyl group is preferred in oxamate (Ox1alt). If we assume that oxamate has a similar intrinsic propensity to an outof-plane carboxyl group worth about $2 \mathrm{kcal} / \mathrm{mol}$ such as for pyruvate, this hydrogen bond energy is roughly $6 \mathrm{kcal} / \mathrm{mol}$, as the total energy difference between 
perpendicular and planar oxamate conformations is about $4 \mathrm{kcal} / \mathrm{mol}$. Like oxamate, the lactate conformational preference is dominated by a hydrogen bond, but in this case, it is between the hydroxyl and carboxyl groups, and is much stronger, with an energy of about $15 \mathrm{kcal} / \mathrm{mol}$ (the difference in energy between lactate with hydroxyl torsions of 0 and $180^{\circ}$ where $\mathrm{O} 6-\mathrm{C} 3-\mathrm{C} 2-\mathrm{O} 7$ is fixed at $0^{\circ}$ ). As rotation of the carboxyl group is more energetically costly than for pyruvate or oxamate because of this hydrogen bond, the only conformer expected to be significantly populated in vacuo is L1, in which the carboxyl and hydroxyl groups are essentially co-planar in a hydrogen-bonded orientation, and with the methyl group staggered with respect to the $\mathrm{sp}^{3}$ carbon $\mathrm{C} 2$.

Diffuse functions in the basis set are clearly necessary to represent these anions adequately, and this observation is particularly true for pyruvate. Qualitatively different conclusions have been obtained here compared with previous calculations at the 3-21G level in which the planar conformation was predicted to be lowest in energy [8]. Calculations employing a larger basis set without diffuse functions $(6-31 \mathrm{G}(\mathrm{d}))$ predict that $\mathrm{P} 1$ and $\mathrm{P} 3$ are isoenergetic (Table 1). Only upon adding diffuse functions do the calculations appear to be converged and consistently predict that the perpendicular conformation is lowest in energy. The necessity for including diffuse functions in this type of molecule was also emphasized in a recent study of oxaloacetate [12], which is similar to pyruvate except that one methyl hydrogen is replaced with a second carboxyl group. These results for oxaloacetate are very similar to our results for pyruvate, with the perpendicular carboxyl conformation $2.4 \mathrm{kcal} / \mathrm{mol}$ more stable than the planar one, compared with the barrier of $2.01 \mathrm{kcal} / \mathrm{mol}$ reported here for pyruvate.

When bound to LDH, however, pyruvate is expected to adopt a near-planar conformation. In the crystallographic structures from Plasmodium falciparum (PDB entry 1LDG [13]), dogfish muscle (1LDM [4]) and Bacillus stearothermophilus (1LDN $[14]$ ), the O6-C3-C2-O7 torsion of the bound inhibitor oxamate is reported to be $-17.4,-27.7$, and $-25.9^{\circ}$, respectively (average $-24^{\circ}$ ). To estimate the energy cost of such a deformation for the three molecules, we calculated single point MP2/6-31+G(d,p) energies for the same geometries as the minimum energy conformers (P3alt, Ox1alt, and L1) except that the carboxyl group torsions were changed to $-24^{\circ}$. As oxamate has a very similar carboxyl group torsion $\left(-19.8^{\circ}\right.$ in vacuo) to that found in the enzyme, the enzyme-bound conformation is only $0.03 \mathrm{kcal} / \mathrm{mol}$ higher in energy than Ox1alt. However, in pyruvate the carboxyl group must rotate from the perpendicular conformation found in vacuo to near-planar upon binding to the enzyme. However, as the barriers to carboxyl rotation are relatively modest, this enzyme-bound conformation is only $1.81 \mathrm{kcal} / \mathrm{mol}$ higher in energy than P3alt. A greater cost for the conformational changes associated with binding is expected for lactate. The difference in energy between L1 and an enzyme-bound conformation (with carboxyl group torsion $-24^{\circ}$ and hydroxyl group torsion $25^{\circ}$ ) is $6.99 \mathrm{kcal} / \mathrm{mol}$. Most of this penalty is attributed to the less favourable intramolecular hydrogen bond geometry. This value can be regarded as an upper limit to the difference in energy, as in the active site the hydroxyl group makes other interactions, which may compensate for the weakened intramolecular hydrogen bond. A lower limit for the energy change upon binding would be provided by the second minimum L2, in which no intramolecular hydrogen bond is present. In the L2 conformation, the carboxyl group torsion $\left(-24.1^{\circ}\right)$ is close to that found for oxamate bound in the enzyme, so no energy would be required for carboxyl rotation upon binding. As L2 is $15 \mathrm{kcal} / \mathrm{mol}$ higher in energy than L1, and the model-bound conformation is $7 \mathrm{kcal} / \mathrm{mol}$ higher in energy than L1, the model-bound conformation is $8 \mathrm{kcal} / \mathrm{mol}$ more favourable than the L2 conformation, a difference thus mostly attributable to the intramolecular hydrogen bond. An estimate of $2-4 \mathrm{kcal} / \mathrm{mol}$ for the energy cost of conformational changes upon binding to the enzyme seems plausible.

These estimates for the differences in energy between the bound and unbound conformations of the substrates are approximate only. In addition to experimental uncertainty in the crystallographic results, the modelling of the enzyme-bound lactate conformation on the crystallographic oxamate geometry is more speculative than for pyruvate. The aqueous solvent is expected to interact strongly with these negatively charged molecules, potentially altering the preferred conformation of the substrates in solution compared with that found in vacuo. In addition, once bound, any energy penalty for carboxyl rotation 
or loss of a hydrogen bond will be counterbalanced by interactions with the enzyme. For example, in the enzyme active site, the charge on the carboxyl group would be expected to be much reduced due to its strong ion-pair interaction with Arg171, which would decrease the repulsion between the carboxyl oxygens and either the carbonyl or hydroxyl oxygen. Likewise, the hydrogen bond between His 195 and the carbonyl or hydroxyl group would influence the energetics of substrate binding. Nonetheless, it is quite interesting that the conformation of the substrate bound in the active site is more similar to the in vacuo conformation of lactate or the inhibitor oxamate than the pyruvate conformation. Pyruvate, however, appears to require less energy to attain the enzyme-bound conformation than does lactate.

Because the potential energy surface which has been calculated for the chemical reaction [3] is of the same order of magnitude as the conformational surfaces presented here, these differences in the conformational flexibility of the substrate pyruvate, product lactate, and the transition state may well influence the chemical reaction. Although the conformation of the methyl group is unlikely to be important, the carboxyl group conformation could be significant in determining the reaction mechanism. Thus, it is crucial that if the semiempirical methods are to be used in the hybrid QM/MM method, they are able to reproduce the conformational preferences. If barriers to these conformational changes are not predicted correctly using the AM1 or PM3 method, the transition state will also be less accurate.

The results indicate that the AM1 method models the substrates reasonably compared with the best ab initio results. The geometries and barriers to carboxyl and methyl rotations are reproduced very well for pyruvate. For lactate, the geometry of the global minimum L1 is reproduced well, but the higher energy region is not modelled as accurately. As this region is $15 \mathrm{kcal} / \mathrm{mol}$ higher in energy than the global minimum, and the conformation of lactate bound in the active site is likely to be similar to the L1 conformation (with a hydroxyl torsion $\mathrm{H} 12-\mathrm{O} 7-\mathrm{C} 2-\mathrm{C} 3$ near $-25^{\circ}$ ), this deficiency of the AM1 method is unlikely to cause problems for $\mathrm{QM} / \mathrm{MM}$ calculations. The AM1 method is less successful in modelling the inhibitor oxamate, however, and predicts a carboxyl torsion of $-65^{\circ}$ which is nearer to perpendicular than the near-planar minimum-energy conformer Ox1alt. In general, the PM3 method tends be less suitable for studying these molecules, primarily due to increased barriers for all torsional rotations. Similar conclusions have been reached on the quality of the results obtained using semiempirical methods for the related molecule oxaloacetate [12].

\section{Conclusions}

The in vacuo conformational preferences of the substrates of LDH have been shown to be dominated by the balance between intramolecular electrostatic repulsion and hydrogen bonds. Pyruvate prefers an out-of-plane carboxyl group orientation, while oxamate (which differs only in the substitution of the methyl group by an amino group) prefers a nearplanar conformation, due to the presence of an intramolecular hydrogen bond. The global minimum for lactate is more similar to that for oxamate, in that the carboxyl group is co-planar with the hydroxyl group. The observed conformation of oxamate bound to the enzyme is near-planar, as for gas-phase oxamate and lactate, but a larger conformational change would be required for pyruvate binding to the enzyme (assuming the solution structure is similar to the gas-phase conformation). As the barrier to carboxyl group rotation is quite low, however, this conformational change requires only $\sim 1.8 \mathrm{kcal} / \mathrm{mol}$ while the predicted lesser conformational change required for lactate to bind might require even more energy because a strong hydrogen bond must be broken when the carboxyl group rotates. This energetic cost upon binding is likely to be modulated by intermolecular interactions between active site residues and the substrate.

The AM1 method models the geometry and relative energies of conformers reasonably well for the substrates pyruvate and lactate. Hybrid QM/MM calculations employing AM1 for the quantum mechanical region are, thus, likely to be adequate in modelling any subtle effects the conformational preferences might have on the chemical reaction.

\section{Supporting information}

The optimized geometry and frequencies for all oxamate, pyruvate, and lactate conformers are 
available (in electronic form) from the authors upon request.

\section{Acknowledgements}

Funding for R.K.S. from an Australian Research Council Postdoctoral Fellowship is gratefully acknowledged, as well as generous computing grants and support from the Australian National University (ANU) Supercomputing Facility. The ANU Strategic Development Fund provided additional funding.

\section{References}

[1] A.R. Clarke, T. Atkinson, J.J. Holbrook, Trends Biochem. Sci. 14 (1989) 101-105.

[2] S. Ranganathan, J.E. Gready, J. Chem. Soc. Faraday Trans. 90 (1994) 2047-2056.

[3] S. Ranganathan, J.E. Gready, J. Phys. Chem. B 101 (1997) 5614-5618.

[4] J.L. White, M.L. Hackert, M. Buehner, M.J. Adams, G.D.
Ford, P.J. Lentz, I.E. Smiley, S.J. Steindel, M.G. Rossmann, J. Mol. Biol. 102 (1976) 759-779.

[5] C. van Alsenoy, L. Schäfer, K. Siam, J.D. Ewbank, J. Mol. Struct. (Theochem) 187 (1989) 271-283.

[6] J. Murto, T. Raaska, H. Kunttu, M. Räsänen, J. Mol. Struct. (Theochem) 200 (1989) 93-101.

[7] P. Tarakeshwar, S. Manogaran, J. Mol. Struct. (Theochem) 430 (1998) 51-56.

[8] K.E. Norris, J.E. Gready, J. Mol. Struct. (Theochem) 258 (1992) 109-138.

[9] K.E. Norris, J.E. Gready, J. Mol. Struct. (Theochem) 279 (1993) 99-125.

[10] T. Clark, J. Chandrasekhar, G. Spitznagel, P.v.R. Schleyer, J. Comput. Chem. 4 (1983) 294-300.

[11] M.J. Frisch et al., Gaussian 94, Revision E.2, Gaussian Inc., Pittsburgh, PA, 1995.

[12] A.J. Mulholland, W.G. Richards, J. Mol. Struct. (Theochem) 429 (1998) 13-21.

[13] C.R. Dunn, M.J. Banfield, J.J. Barker, V.E. Higham, K.M. Moreton, D. Turgut-Balik, R.L. Brady, J.J. Holbrook, Nat. Struct. Biol. 3 (1996) 912-915.

[14] D.B. Wigley, S.J. Gamblin, J.P. Turkenburg, E.J. Dodson, K. Piontek, H. Muirhead, J.J. Holbrook, J. Mol. Biol. 223 (1992) 317-335. 